\title{
Estudo da estrutura interna do Teste de Inteligência - TI
}

\author{
Monalisa Muniz Nascimento- Universidade Federal de São Carlos, São Carlos, Brasil \\ Fabián Javier Marin Rueda - Universidade São Francisco, Itatiba, Brasil
}

\begin{abstract}
Resumo
O objetivo do trabalho foi verificar a estrutura interna do Teste de Inteligência -TI, por meio da Análise Fatorial Exploratória - AFE e Confirmatória - AFC. A amostra para a pesquisa se constituiu em 2.163 sujeitos, sendo 1.013 para o estudo da AFE e 1150 para a AFC. Em ambos os casos os sujeitos foram maiores de 18 anos, de ambos os sexos, e com escolaridade variando de ensino fundamental incompleto até o ensino superior completo. Inicialmente fez-se a AFE, que sugeriu a presença de dois fatores, o primeiro englobando os itens 18 a 30 e o segundo formado pelos itens de 1 a 17, mas que se correlacionaram de forma positiva e moderada. Diante desse resultado aplicou-se a AFC, que também mostrou o modelo com dois fatores como sendo o mais ajustado. Apesar dos resultados apontarem para uma estrutura bidimensional, mais estudos são necessários para melhor compreensão dos fatores.

Palavras-chave: Evidência de validade; Análise fatorial; Raciocínio; Construção de instrumentos; Psicometria.
\end{abstract}

\section{Internal structure of the TI - Teste de Inteligência}

\begin{abstract}
This study aimed at verifying the internal structure of the Intelligence Test - TI using exploratory factor analysis (EFA) and confirmatory factor analysis (CFA). The sample was composed of 2163 subjects, 1013 of those to the EFA study and 1150 to CFA. In both cases, the subjects were over 18 years old, both males and females, with school level ranging from elementary school to college. Initially an EFA was run and suggested two factors, the first one including items 18 to 30 , and the second one including items 1 to 17, but with a moderate and positive correlation between factors. After this result, the AFC was run, which also suggested a two-factor model as the most adequate. Despite results showing a bi-dimensional structure, more studies are necessary for a better understand the internal structure of the TI.

Keywords: Validity evidence, Factor analysis, Reasoning, Psychometrics, Instrument development.
\end{abstract}

Estudio de la estructura interna del Teste de Inteligência -TI

\begin{abstract}
Resumen
El objetivo del trabajo fue verificar la estructura interna del Teste de Inteligência - TI, a través del Análisis Factorial Exploratorio -AFE y Confirmatorio - AFC. La muestra de la investigación se constituyó por 2163 sujetos, siendo 1013 para el estudio del AFE y 1150 para el AFC. En los dos casos los sujetos eran mayores de 18 años, ambos sexos, y con escolaridad variando desde la educación primaria incompleta hasta la enseñanza superior completa. Inicialmente se realizó el AFE, que sugirió la presencia de dos factores, el primero incluyendo los ítems 18 a 30 y el segundo formado por los ítems de 1 a 17, pero que presentaron una correlación positiva y moderada. Frente a ese resultado se aplicó el AFC, que también mostró que el modelo con dos factores fue el más ajustado. Pese a que los resultados apuntan para la existencia de una estructura bidimensional, más estudios son necesarios para entender mejor esos factores.

Palabras-clave: Evidencia de validez; Análisis factorial; Raciocinio; Construcción de instrumentos; Psicometría.
\end{abstract}

A inteligência é um construto que três grandes teorias procuram explicar sob diferentes perspectivas, quais sejam, a teoria desenvolvimentista, a psicologia cognitiva ou abordagem do processamento humano da informação e a psicométrica (Almeida, 1988, 1994; Sternberg, 1986). $\mathrm{O}$ primeiro modelo compreende a inteligência como um fenômeno que se desenvolve por etapas, de forma crescente, invariante na sua estruturação, e de forma qualitativamente diferente entre os indivíduos. $\mathrm{O}$ método investigativo utilizado nessa teoria é a observação e a descrição dos fenômenos, como por exemplo, a fala, a interação com o ambiente e a aprendizagem.
$\mathrm{Na}$ concepção da psicologia cognitiva, o termo inteligência abrange e unifica as dimensões cognitivas, objetivando sistematizar os processos mentais subjacentes à cognição, como a sensação, a percepção e a memória. Essa teoria estuda os processos cognitivos envolvidos na resolução de tarefas, como, por exemplo, em situações nas quais é necessário comparar informações, o indivíduo tende a mapear e codificar os elementos, utilizando a atenção para selecionar o que será analisado, guardando na memória diversos estímulos para fazer uma análise comparativa das informações, elaborar uma resposta e julgar se a mesma está correta (Muniz, em preparação). 
Por sua vez, o modelo psicométrico investiga a estrutura e a organização subjacente à inteligência, utilizando-se frequentemente do método de análise fatorial. O objetivo da técnica fatorial é identificar a estrutura subjacente em um construto, por meio de agrupamentos de variáveis. Assim, pode-se identificar se determinado construto é composto por um ou mais fatores. A teoria psicométrica, junto à análise fatorial, possibilitou a compreensão da inteligência como multidimensional, com diversos fatores, especificando os tipos de inteligência que o ser humano pode apresentar.

Mais recentemente, outra abordagem está se consolidando no estudo da inteligência, a saber, a neurociência cognitiva. A finalidade dessa abordagem é a identificação dos processos cognitivos da inteligência, relacionando-os com os aspectos estruturais e funcionais do cérebro (Hunt, 1999; Posner \& DiGiloramo, 2000). Com isso, já se sabe que o córtex pré-frontal é o principal local de processamento das informações para o raciocínio, principalmente porque é onde se encontra a base neurológica das funções executivas. Essas funções estão entre os aspectos mais complexos da cognição e envolvem a seleção e integração de informações atuais com informações previamente memorizadas, $\mathrm{o}$ planejamento, o monitoramento e a flexibilidade cognitiva (Gazzaniga, Ivry, Mangun 2002; Lezak, 1995) e, consequentemente, são funções que interferem na aprendizagem.

Importante mencionar que todas essas teorias contribuem para o entendimento da inteligência, sendo complementares e não excludentes. No processo de construção de instrumento psicológico para avaliação da inteligência na sua fundamentação, a maior parte das vezes está presente a teoria psicométrica. Para o Teste de Inteligência - TI (Rueda \& Castro, 2012) não foi diferente, pois os autores embasaram o instrumento na teoria de Charles Spearman, e o objetivo é mensurar a capacidade de edução de relação, considerada como um dos elementos do fator $g$ ou inteligência geral proposto por Spearman $(1904,1927)$.

As teorias fatoriais da inteligência podem ser divididas entre a tradição britânica e a tradição americana. A britânica tem como destaque a teoria de Spearman e a americana é representada por Thurstone. Spearman investigou se as habilidades intelectuais se correlacionavam entre si e se eram dependentes ou independentes de um fator geral, comum a todas elas. Para isso correlacionou diversos testes que mensuravam habilidades distintas e identificou correlações entre eles, inclusive altas, o que o levou a inferir a existência de um fator comum, geral entre as habilidades. Além disso, por meio do método de análise fatorial desenvolvido por ele, pode observar a extração de dois fatores relacionados entre si. O primeiro foi denominado de geral, comum entre todas as habilidades; e o segundo denominado específico, algo que cada habilidade possui em particular. Surgiu assim a Teoria Bifatorial de Spearman, concebendo dois fatores. Tal teoria pressupõe que, para a realização de qualquer atividade intelectual, é necessário o fator geral e um fator específico condizente com a tarefa proposta. No entanto, o fator específico não é totalmente independente, pois possui correlação com o geral. Após vários estudos, Spearman verificou um terceiro fator, o de grupo, que é formado por habilidades semelhantes. Dessa forma, a teoria de Spearman passou a ser conhecida como trifatorial, composta pelo fator geral $-g$, fatores específicos $-e$ e de grupo (Spearman, 1927).

Cognitivamente, o fator $g$ pode ser descrito por três processos básicos. $O$ primeiro seria a apreensão da experiência, que se refere à capacidade relacionada à percepção, rapidez e acuidade para discriminar os estímulos e apresentar consciência desse processo. $\mathrm{O}$ segundo, edução das relações, é a capacidade do indivíduo em detectar relações entre duas ou mais ideias percebidas no ambiente ou evocadas da memória. Já o terceiro processo, denominado de edução de correlatos, refere-se à capacidade de formular novas ideias ou relações a partir de ideias ou relações já estabelecidas (Spearman, 1927).

A tradição americana das teorias fatoriais, inicialmente, se contradizia com a tradição britânica, no que se refere à existência de um fator geral. Thurstone (1934), a partir de uma análise fatorial para verificar habilidades da inteligência, formulou a Teoria das Aptidões Primárias, independentes entre si e sem um fator comum entre elas. Tais fatores são a compreensão verbal, a fluência verbal, o raciocínio indutivo, numérico, aritmético e dedutivo, a visualização espacial, a memória e a rapidez perceptiva.

A divergência inicial entre as teorias de Spearman e Thurstone levou outros pesquisadores a estudar a estrutura do construto inteligência, por meio da análise fatorial. Então, na década de 1950, surgiram alguns modelos tentando conciliar as duas teorias (Almeida, 1988). Segundo Sisto (2006), ainda há pesquisadores que apoiam a Teoria de Thurstone sobre as Aptidões Primárias, porém o próprio Thurstone (Thurstone \& Thurstone, 1941 citado por Sisto, 2006), por meio de outros trabalhos, verificou a existência de um fator geral 
da inteligência, concordando com Spearman. Hoje em dia a teoria psicométrica mais moderna e difundida é a do modelo Cattell-Horn-Carroll (CHC) das habilidades cognitivas, que integra essas duas tradições (Carroll, 1997).

A teoria $\mathrm{CHC}$ concebe a inteligência como multidimensional e composta por áreas amplas do funcionamento cognitivo, mas relacionadas a um fator geral. Três estratos representam a teoria CHC. O primeiro são as habilidades específicas, que estão ligadas ao segundo estrato, que contempla as capacidades. Essas capacidades, por sua vez, estão conectadas ao terceiro extrato, que é o fator comum, geral a todas essas habilidades e capacidades. Desse conjunto de capacidades, dez fazem parte desde o início do modelo CHC (inteligência/raciocínio fluido, inteligência/raciocínio cristalizado, raciocínio/conhecimento quantitativo, leitura-escrita, memória de curto prazo, inteligência/ processamento visual, inteligência/processamento auditivo, armazenamento e recuperação associativa de longo prazo, rapidez de processamento cognitivo, e tempo/velocidade de decisão/reação), e outras seis foram incorporadas ao modelo, mais especificamente a partir do ano de 2009, com uma publicação de McGrew (conhecimento geral/domínio específico, velocidade psicomotora, habilidade psicomotora, habilidade olfativa, habilidade tátil, e habilidade cinestésica). Essas seis últimas capacidades não estão bem estabelecidas, porém foram reafirmadas no artigo de Schneider e McGrew (2011), que aborda atualidades da teoria CHC, e com elas pesquisas estão sendo desenvolvidas.

No que se refere à avaliação do fator $g$, apenas ocorre por meio da mensuração dos fatores/capacidades específicas, mas mesmo assim, não há como medi-lo "totalmente", pois não há testes psicológicos para mensurar todas as capacidades, bem como pode-se pensar que ainda não sejam conhecidas, de fato, todas as que realmente existem. No entanto, segundo Alves (2002), testes não verbais, homogêneos e de edução de relações e correlatos, parecem ser mais indicados para a avaliação do fator $g$, pois apresentariam maior relação com esse fator. O Teste de Inteligência - TI (Rueda \& Castro, 2012) tem a finalidade de avaliar essas capacidades (Spearman, 1904, 1927), assim como o teste das Matrizes Progressivas de Raven - MPR (Raven, 2000), o teste das Matrizes Progressivas Coloridas de Raven MPCR (Angelini e cols., 1999) e o Teste Não Verbal de Inteligência - R1 (Alves, 2002), todos com evidência de validade para a população brasileira.
Para que o processo edutivo seja operacionalizado nos testes que se baseiam na teoria de Spearman, constroem-se estímulos não verbais, para que se identifique uma relação já estabelecida entre eles. As tarefas elaboradas podem ser de complementação de figura concreta ou abstrata, identidade, alternância de elementos, dentre outros (Alves, 2002; Angelini e cols, 1999).

O Teste de Inteligência (TI), objeto do presente estudo, é formado por itens com figuras abstratas que solicitam a complementação de fundos geométricos. Para resolver a tarefa, o sujeito precisa escolher, dentre as alternativas oferecidas, aquela que complete adequadamente o fundo geométrico. Tarefas dessa natureza também podem ser encontradas nas MPR, MPCR e R1.

Os testes mencionados e baseados na teoria de Spearman pressupõem unidimensionalidade, pois estariam avaliando o fator geral da inteligência. No entanto, é preciso que seja demonstrado empiricamente que esses testes podem ser interpretados de forma unidimensional. Nesse sentido, uma das evidências de validade das interpretações de um teste psicológico é a estrutura interna, que indica o nível em que as relações entre os itens do teste e/ou seus componentes ou subtestes são coerentes com a estrutura de relações proposta pela definição teórica. A estrutura conceitual de um teste pode implicar uma ou mais dimensões, assim como também pode pressupor uma estrutura hierárquica ou de fatores isolados. Nesses casos, essa forma de evidência verifica a coesão entre a estrutura prevista com a observada. Nessa categoria também inclui-se a análise da estrutura das relações entre os itens de um teste predestinados, por teoria, a avaliar um mesmo construto (AERA, APA, NCME, 1999).

Algumas pesquisas procuraram investigar a unidimensionalidade das MPCR e do R1-Forma B. Pasquali, Wechsler e Bensusan (2002), utilizando uma amostra de 9.929 sujeitos com idade entre 5 e 11 anos, efetuaram uma análise de estrutura interna pela Teoria Clássica dos Itens com as MPCR. A análise fatorial identificou quatro fatores, que foram denominados de raciocínio analógico-abstrato, raciocínio analógico-concreto, percepção da gestalt e raciocínio dedutivo. Os quatro fatores apresentaram correlação e juntos formaram um fator geral identificado como raciocínio analógico, contendo 25 dos 36 itens do teste original, mas, segundo os autores, somente o primeiro fator e o geral possuíam itens suficientes e com boas cargas fatoriais para representação comportamental do construto.

Também sobre as MPCR, Sisto, Rueda e Batholomeu (2006) avaliaram a estrutura do teste de acordo 
com o modelo Rasch. Participaram 441 crianças de $1^{\text {a }}$ a $4^{a}$ série do ensino público municipal de uma escola do interior do estado de São Paulo, e as idades variavam de 7 a 11 anos. Para as análises, utilizou-se a forma geral das MPCR, assim como cada série (A, Ab e B) de forma separada. Os resultados indicaram que somente a série $A b$ é unidimensional. No teste geral e na série $\mathrm{B}$, os autores concluíram pela não unidimensionalidade, enquanto na série A, sugeriram haver incerteza sobre a existência de apenas um fator.

Para o teste R1-Forma B, Sisto, Santos e Noronha (2007), a partir de uma amostra com 745 estudantes com idades entre 15 e 75, aplicaram a análise fatorial, por meio do modelo de Rasch, e a unidimensionalidade não foi observada. Os resultados indicaram a presença de um segundo fator, o que condiz com os estudos do manual desse teste, que foi elaborado por Sisto, Santos e Noronha (2004). Os mesmos autores, no manual do teste, indicaram a existência de dois fatores, mas com um fator comum subjacente. Nesse sentido, ponderaram que a interpretação dos dados somente por um fator não seria suficiente para avaliar as habilidades cognitivas envolvidas na realização do teste.

Os estudos citados mostram a importância de se investigar e conhecer a estrutura do construto que é avaliado em um teste psicológico. $\mathrm{O}$ embasamento teórico para a construção dos itens é fundamental, mas é preciso que empiricamente seja testada a teoria proposta. O Teste de Inteligência - TI (Rueda \& Castro, 2012) foi construído para ser unidimensional, seguindo a proposta teórica de Spearman, mas apesar de várias evidências de validade já constatadas e presentes em seu manual, até o momento não há estudos sobre a estrutura interna do teste, com base na análise fatorial. Diante disso, o presente artigo tem como objetivo investigar essa estrutura por meio da análise fatorial exploratória (AFE) e confirmatória (AFC).

\section{Método}

\section{Participantes}

Para a realização do estudo, contou-se com uma amostra de 2.163 sujeitos. Na realização da AFE, utilizou-se um banco de dados composto por 1.013 sujeitos, que responderam ao TI no ano de 2011, nos estados de São Paulo (75,1\%) e Mato Grosso do Sul (24,9\%), em clínicas de avaliação psicológica para $\mathrm{CNH}$ credenciadas aos DETRANs dos respectivos estados. A idade variou de 18 a 65 anos $(M=26,28 ; D P=9,16)$, e $58,8 \%$ eram do sexo masculino. Em relação à escolaridade, 26,9\% possuíam ensino fundamental completo ou incompleto, $56,6 \%$ o ensino médio completo ou incompleto e $16,5 \%$, ensino superior completo ou incompleto.

No que se refere ao estudo por meio da AFC, a amostra foi de 1.150 sujeitos, que responderam ao teste no ano de 2012, e provenientes de clínicas de avaliação psicológica para $\mathrm{CNH}$ dos estados da Bahia (27,8\%), São Paulo (22,7\%), Mato Grosso do Sul $(19,2 \%)$ e Amapá (30,3\%). A idade variou de 18 a 73 anos, com média de 27,70 anos $(D P=9,46)$. A escolaridade variou entre ensino fundamental completo ou incompleto $(10,3 \%)$, ensino médio completo ou incompleto $(56,4 \%)$ e ensino superior completo ou incompleto $(33,3 \%)$. Em relação ao sexo, 65\% eram do masculino.

\section{Instrumento}

Teste de Inteligência - TI (Rueda \& Castro, 2012).

O TI foi construído para mensurar a capacidade de edução de relações, um dos elementos da inteligência geral proposta por Spearman $(1904,1927)$. O teste é formado por 30, itens compostos por figuras geométricas nas quais o avaliando precisa identificar a parte que falta da figura. Há seis opções de alternativas e somente uma é correta. Cada acerto equivale a 1 ponto e o tempo para resolução é de 20 minutos. É um teste destinado para indivíduos entre 18 e 65 anos e pode ser aplicado de forma individual ou coletiva.

No manual são apresentados alguns estudos sobre as propriedades psicométricas do instrumento. A estrutura interna foi estudada pelo funcionamento diferencial do item considerando o sexo das pessoas e a unidade federativa na qual residiam. Nesse sentido, não foi encontrada diferença no desempenho em função do sexo, enquanto 12 dos 30 itens apresentaram DIF em razão da unidade federativa dos sujeitos, o que motivou a elaboração de normas específicas para os estados analisados.

Ainda, o TI foi estudado com medidas como a idade, a escolaridade, e os testes R1 e AC. No que se refere ao primeiro estudo, a correlação de Pearson indicou coeficientes negativos e significativos $(r=-0,43$, $p<0,001)$, evidenciando que à medida que a idade aumenta, há um decréscimo nas pontuações do TI. Já em relação à variável escolaridade, foi observada uma correlação positiva e significativa $(r=0,35, p<0,001)$. Com isso, pode-se compreender que um nível de inteligência superior pode estar associado ao aumento da escolaridade. 
No que tange à comparação com outros testes, a correlação de Pearson com o teste R1 forneceu evidência de validade convergente para o TI, uma vez que o coeficiente obtido foi 0,56. Por fim, o estudo com o AC mostrou uma correlação de 0,38 , indicando a evidência de validade para o TI por meio da comparação com testes que avaliam construtos relacionados.

Em relação aos estudos de precisão, foi estudada a consistência interna por meio do coeficiente alfa de Cronbach e o método das duas metades de Spearman-Brown e Guttman. Os coeficientes obtidos variaram entre 0,90 e 0,93 , indicando que a quantidade de erro presente nos resultados advindos do TI é muito pequena.

\section{Procedimento}

Inicialmente o projeto foi submetido a um Comitê de Ética em Pesquisa, e, após sua aprovação, os dados foram coletados em clínicas de avaliação psicológica credenciadas aos Departamentos Estaduais de Trânsito dos Estados da Bahia, Mato Grosso do Sul, São Paulo e Amapá. Todos os instrumentos foram aplicados por psicólogos peritos credenciados, de forma individual e após assinatura do Termo de Consentimento Livre e Esclarecido. A duração aproximada da aplicação foi de 30 minutos.

\section{Análise dos dados}

Para a investigação da evidência de estrutura interna sobre a dimensionalidade do TI, inicialmente fez-se uma análise fatorial exploratória utilizando o programa TestFact, por meio da análise Full Information Factor Análises (FIFA), que faz uso da TRI e coeficientes de correlação tetracórica. Como critério de análise da estrutura encontrada, verificou-se a divisão entre o eigenvalue do primeiro e segundo fator assumindo valores acima de 5 como sugestão de unidimensionalidade e carga fatorial dos itens acima de 0,30 (Bock, Gibbons \& Muraki, 1988; Primi \& Almeida, 1998).

$\mathrm{Na}$ segunda investigação da dimensionalidade, optou-se por efetuar a análise fatorial confirmatória com o programa estatístico MPLUS 7. A estimação foi efetuada pelo método dos mínimos quadrados ponderados robustos - WLSMV (Muthén \& Muthén, 2004). Para avaliar o ajuste dos modelos, optou-se por utilizar cinco índices, quais sejam, a magnitude do qui-quadrado - $X^{2} / g l$, o índice de ajuste comparativo (CFI), o índice Tucker-Lewis (TLI), os resíduos raiz do erro médio quadrático de aproximação (RMSEA) e o weighted root mean square residual (WRMR). De acordo com autores como
Arbuckle (2009), Bentler e Bonnet (1980), e Tabachnik e Fidell (2001), os valores aceitáveis para cada um desses índices é diferente. No caso do $\mathrm{X}^{2} / g l$, são considerados excelentes valores entre 1 e 2 , muito bons até 3 , aceitáveis entre 3 e 5 (considerando principalmente se as análises são realizadas com amostras grandes) e rejeitados acima de 5. Para o CFI e TLI, são considerados adequados valores acima de 0,90 , enquanto para o RMSEA, valores até 0,08 indicam que o modelo se ajusta apropriadamente, embora até 0,05 sejam considerados ótimos. Por fim, o valor de WRMR deve estar abaixo de 1,0 para ser considerado ótimo.

$\mathrm{Na}$ AFC, os modelos testados foram de 1 fator, de acordo com a teoria da inteligência geral de Spearman e também pressuposto no manual do TI, e o de 2 fatores, conforme encontrado nas análises por meio do FIFA. Além das análises mencionadas, inicialmente foram realizadas estatísticas descritivas de média e desvio padrão referentes ao desempenho da amostra no TI, além de frequências e porcentagens para a compilação dos dados da amostra por sexo, escolaridade e estado.

\section{Resultados}

A pontuação no TI para a primeira amostra atingiu a média de 15,52 e desvio padrão 6,91, enquanto para a segunda amostra, a média foi de 14,43 e desvio padrão 6,95. Essas informações são semelhantes aos dados do manual, que apresentam média 15 e desvio padrão de 6,69 para a amostra estudada na normatização do teste.

Realizando a análise FIFA, extraiu-se o modelo de um e dois fatores, com saturação acima de 0,30. O primeiro modelo explicou $42,35 \%$ da variância e o segundo, $54,73 \%$, sendo $39,41 \%$ do fator 1 e 15,32 do fator 2 . Na Tabela 1 podem-se visualizar as cargas fatoriais, a comunalidade e a variância explicada na análise dos dois modelos.

Por meio da Tabela 1 observa-se que todos os itens foram mantidos com o critério de saturação a partir de 0,30. O modelo com um fator apresenta dados que tendem a indicar a unidimensionalidade do instrumento, pois obteve uma variância explicada considerável, além de todos os itens mostrarem cargas fatoriais acima de 0,30 , com a maioria superior a 0,50 . No entanto, a divisão entre o eigenvalue do primeiro fator $(13,07)$ e do segundo fator apontado $(4,22)$ foi 3,10 , não atingindo o valor 5 como critério de unidimensionalidade (Bock, Gibbons \& Muraki, 1988; Primi \& Almeida, 1998). Além disso, notou-se que, a partir do item 18, as cargas fatoriais, exceto do item 29, foram entre 0,72 e 0,86 , consideradas muito altas. Com base nessas informações, 
Tabela 1. Modelo com um e dois fatores, cargas fatoriais, variância explicada e índice de comunalidade pela análise FIFA

Teste de Inteligência - TI

\begin{tabular}{|c|c|c|c|c|c|c|}
\hline \multicolumn{3}{|c|}{1 fator } & \multicolumn{4}{|c|}{2 fatores } \\
\hline Item & 1 & $h^{2}$ & Item & 1 & 2 & $h^{2}$ \\
\hline 1 & 0,31 & 0,09 & 1 & & 0,48 & 0,19 \\
\hline 2 & 0,41 & 0,20 & 2 & & 0,54 & 0,29 \\
\hline 3 & 0,40 & 0,16 & 3 & & 0,59 & 0,30 \\
\hline 4 & 0,52 & 0,27 & 4 & & 0,59 & 0,35 \\
\hline 5 & 0,43 & 0,18 & 5 & & 0,46 & 0,23 \\
\hline 6 & 0,56 & 0,32 & 6 & & 0,60 & 0,38 \\
\hline 7 & 0,50 & 0,25 & 7 & & 0,73 & 0,45 \\
\hline 8 & 0,60 & 0,36 & 8 & & 0,88 & 0,65 \\
\hline 9 & 0,49 & 0,24 & 9 & & 0,72 & 0,43 \\
\hline 10 & 0,58 & 0,39 & 10 & & 0,83 & 0,59 \\
\hline 11 & 0,69 & 0,48 & 11 & & 0,88 & 0,72 \\
\hline 12 & 0,56 & 0,31 & 12 & & 0,76 & 0,52 \\
\hline 13 & 0,55 & 0,30 & 13 & & 0,77 & 0,53 \\
\hline 14 & 0,59 & 0,35 & 14 & & 0,45 & 0,37 \\
\hline 15 & 0,37 & 0,13 & 15 & & 0,24 & 0,14 \\
\hline 16 & 0,68 & 0,46 & 16 & 0,35 & 0,45 & 0,47 \\
\hline 17 & 0,60 & 0,36 & 17 & 0,32 & 0,38 & 0,36 \\
\hline 18 & 0,80 & 0,63 & 18 & 0,55 & 0,40 & 0,66 \\
\hline 19 & 0,79 & 0,63 & 19 & 0,64 & & 0,65 \\
\hline 20 & 0,78 & 0,60 & 20 & 0,52 & 0,38 & 0,60 \\
\hline 21 & 0,86 & 0,73 & 21 & 0,71 & & 0,73 \\
\hline 22 & 0,86 & 0,73 & 22 & 0,84 & & 0,78 \\
\hline 23 & 0,77 & 0,59 & 23 & 0,79 & & 0,65 \\
\hline 24 & 0,72 & 0,52 & 24 & 0,82 & & 0,64 \\
\hline 25 & 0,80 & 0,64 & 25 & 0,97 & & 0,83 \\
\hline 26 & 0,76 & 0,58 & 26 & 0,99 & & 0,84 \\
\hline 27 & 0,78 & 0,60 & 27 & 0,98 & & 0,83 \\
\hline 28 & 0,73 & 0,54 & 28 & 0,99 & & 0,82 \\
\hline 29 & 0,61 & 0,58 & 29 & 0,90 & & 0,73 \\
\hline 30 & 0,73 & 0,53 & 30 & 0,90 & & 0,71 \\
\hline \multicolumn{3}{|c|}{ Variância 42,35\% } & \multicolumn{4}{|c|}{ Variância total $54,73 \%$} \\
\hline
\end{tabular}


decidiu-se explorar melhor a estrutura, optando por um modelo com dois fatores.

A extração desse segundo modelo, com dois fatores, obteve uma variância explicada de 54,73\% e cargas fatoriais mais robustas nos dois fatores emergidos. Apesar dos itens 16, 17 e 20 terem carregado nos dois fatores, é nítida a separação entre eles, ficando o fator 2 composto pelos itens de 1 a 17 e o fator 1 do item 18 ao 30. Outra informação importante é a correlação entre os dois fatores, que foi moderada $(r=0,47)$. Essas análises iniciais parecem sugerir que o TI não é unidimensional, mas apresenta duas dimensões que se correlacionam. Procurando obter mais informações sobre a dimensionalidade, optou-se por testar esses dois modelos, com um e dois fatores, por meio da análise fatorial confirmatória. A Tabela 2 mostra os índices de ajustes encontrados para o modelo uni e bifatorial, por meio do programa estatístico MPLUS 7.

Analisando os índices de ajustes dos dois modelos testados, verifica-se que o de dois fatores apresentou resultados mais adequados. O CFI, TLI, RMSEA e $X^{2} /$ $g l$ atingiram os valores aceitáveis, mas o WRMR deveria estar abaixo de 1,0 para ser considerado ótimo; mesmo assim, foi bem menor no modelo bifatorial, sugerindo melhor ajuste para esse modelo.

\section{Discussão}

Analisando os dados obtidos na análise fatorial exploratória e confirmatória, estes sugerem bidimensionalidade para o TI. Na AFE a extração com dois fatores apresentou maior variância explicada, maiores cargas fatoriais e comunalidade dos itens. Além disso, a correlação observada entre os dois fatores $(r=0,47)$ é considerada de magnitude moderada, pressupondo que são fatores relacionados, agregando variância comum do construto mensurado pelo TI.

É importante destacar que os fatores ficaram compostos pela divisão dos itens iniciais e finais, sendo o fator 1 formado pelos itens de 18 a 30 e o fator 2 abrangendo os itens de 1 a 17 . No entanto, observa-se que os itens $16,17,18,19$ e 20 carregaram nos dois fatores, o 16 e 17 com maior carga no fator 2 e os itens 18, 19 e 20, no fator 1 . Também se devem considerar as cargas fatoriais muito elevadas no fator 1 , que do item 22 ao 30 atingiram valores entre 0,79 e 0,99 .

Todos os itens do teste foram desenvolvidos com o mesmo padrão, pois são figuras compostas por desenhos geométricos nos quais falta uma parte que deve ser completada, sendo a tarefa do sujeito perceber entre seis alternativas qual corresponde ao pedaço que preenche a figura. Visualmente não há características nos desenhos que sugiram maior ou menor dificuldade nos itens ou formas diferenciadas de raciocinar para a resolução do problema. A extração dos dois fatores, e que também foi o modelo mais ajustável, parece desvinculado a tipos de itens diferentes.

Uma possível explicação para os dois fatores encontrados pode estar associada à resolução completa do teste. O limite de tempo para solucionar os 30 itens é de 20 minutos, então os sujeitos tendem a não conseguir responder todos os itens, bem como também podem finalizar o teste, mas de forma rápida, sem analisar adequadamente os itens finais, somente para alcançar o término do teste. Dessa forma, um fator pode ter se configurado com os itens iniciais e que as pessoas respondem com a possibilidade de maior tempo e raciocinando mais, enquanto o outro fator pode ter se formado pelos itens finais, com menos probabilidade do sujeito conseguir responder.

Os dados deste estudo estão de acordo com os apresentados por outros trabalhos que procuraram investigar a dimensionalidade de testes de inteligência, também embasados na teoria de Spearman. Em estudos com as MPCR, teste composto por itens com desenhos geométricos em que o sujeito também precisa completar uma parte que falta, os autores Pasquali, Wechsler e Bensusan (2002) encontraram quatro fatores correlacionados e que juntos formavam um fator geral, mas abrangendo somente 25 dos 36 itens do teste original.

A estrutura fatorial verificada por meio da AFE do presente estudo também mostra uma estrutura unidimensional interessante, pois todos os itens carregam com cargas fatoriais aceitáveis, mas menos adequadas

Tabela 2. Índices de ajustes dos modelos testados no MPLUS

\begin{tabular}{lccccc}
\hline Modelo & $X^{2} / \mathrm{gl}$ & RMSEA & CFI & TLI & WRMR \\
\hline 1 fator & 12,56 & 0,100 & 0,837 & 0,825 & 3,531 \\
2 fatores & 5 & 0,059 & 0,944 & 0,939 & 2,161 \\
\hline
\end{tabular}


que o modelo de dois fatores, que apresenta maior variância explicada e itens com cargas fatoriais maiores. Além disso, a AFC também sugere uma estrutura bidimensional, mostrando que é sempre importante testar as estruturas da AFE, ainda mais quando há poucos estudos de validade fatorial, como é o caso do TI e das MPCR.

Por sua vez, o trabalho de Sisto, Rueda e Bartholomeu (2006) também não evidenciou unidimensionalidade para as MPCR. Vale destacar que as MPCR analisadas neste estudo diferem da versão utilizada na pesquisa de Pasquali, Wechsler e Bensusan (2002), que seguiu a versão espanhola, mas são praticamente idênticas, pois a utilizada em 2006 apresenta somente a mudança na sequência dos itens 11 e 12 . Diferentemente das MPCR, o TI é um teste novo, de 2012, e até o momento não havia pesquisa sobre sua estrutura. Diante dos resultados das MPCR, que é um teste muito consolidado sobre a educão de relações, nota-se a dificuldade em estabelecer a unidimensionalidade em testes de inteligência com essas características.

Outro estudo, que também corrobora os dados encontrados na presente pesquisa e os trabalhos efetuados com as MPCR, foi o desenvolvido por Sisto, Santos e Noronha (2007), com o Teste R1, que é um teste de inteligência também baseado na teoria de Spearman em que os autores, aplicando a unidimensionalidade pelo modelo de Rasch, verificaram uma estrutura com dois fatores, que se mostrou mais adequada. Além de ser mais um teste fundamentado na teoria de Spearman e que abrange itens formulados com constituição semelhante às MPCR e TI, a consideração dos autores sobre a inadequação de interpretar os dados pela unidimensionalidade sinaliza a justificativa mais importante para se investigar a estrutura de um teste.

Os dados encontrados nas pesquisas com as MPCR, R1 e agora com o TI mostram o quanto é importante identificar a estrutura de um teste, pois o estudo da dimensionalidade possibilita verificar quais construtos estão sendo mensurados, mesmo que sejam correlacionados a um construto maior. No caso do TI, apesar da estrutura com dois fatores ser a mais promissora, ainda devem-se efetuar mais estudos para a compreensão desse resultado, pois teoricamente, os itens podem ser concebidos pertencentes a um fator, e que é referente ao construto que o TI propõe avaliar, a edução de relação, um dos elementos do fator $g$ (Spearman, 1904, 1927). Além disso, a correlação encontrada entre os dois fatores pode ser um índice estatístico para aumentar a suspeita da unidimensionalidade.

\section{Considerações finais}

Considerando que um conjunto de itens é utilizado para avaliar um determinado construto, e que ao ser interpretado ele indicará certa intensidade do traço medido na pessoa, se torna imprescindível que no teste psicológico esteja especificado qual ou quais construtos podem ser interpretados por essa ferramenta. De acordo com a AERA, APA, NCME (1999), a análise fatorial possibilita investigar a estrutura interna do teste psicológico e as relações entre os itens, comparando-os com a teoria que fundamenta o instrumento. No caso do TI, a suposição seria somente o construto edução de relações, conforme a teoria de Spearman (1904, 1927), tanto que a interpretação do teste é baseada em um escore geral, que é a somatória de acertos em todos os itens realizados pelo sujeito. Analisando o conteúdo dos itens do TI e a forma como foram construídos, todos bastante semelhantes e exigindo que o sujeito relacione informações para encontrar a resposta correta, percebe-se que o construto em questão realmente é avaliado no teste. No entanto, as análises apontam que uma estrutura com dois fatores seria mais adequada, então implica-se a necessidade de outras pesquisas para melhor compreender a estrutura do TI.

De fato, futuros estudos poderiam analisar a estrutura TI considerando até o item no qual a pessoa alcançou ao término dos 20 minutos. Esse aspecto pode ser aventado como uma das possibilidades para a existência do segundo fator, uma vez que ao realizar uma análise dos protocolos respondidos, a maioria conseguiu concluir até o exercício 20 aproximadamente, mas como a correção do instrumento é feita por meio do acerto ( 1 ponto) e erro (0 ponto), a não conclusão de alguns itens, devido à falta de tempo, fez com que os mesmos fossem considerados erros do sujeitos.

O objetivo do trabalho foi um estudo inicial sobre a estrutura do TI, mas a contribuição dessa pesquisa deve ser compreendida além da estrutura para um teste. É importante que se perceba que os estudos fatoriais apresentam um papel importante na construção e validação dos testes psicológicos, pois conhecer as dimensões que podem ser mensuradas com o instrumento aumenta a efetividade e o benefício do uso do instrumento.

\section{Referências}

Almeida, L. S. (1988). Teorias da Inteligência. Porto: Edições Jornal de Psicologia. 
Almeida, L. S. (1994) Inteligência: definição e medida. Aveiro: CIDInE.

Almeida, L. S., Nascimento, E., Lima, A. O. F., Vasconcelos, A. G., Akama, C. T., \& Santos, M. T. (2010). Bateria de Provas de Raciocínio (BPR-5): estudo exploratório em alunos universitários. Avaliação Psicológica, 9(2), 155-162.

Alves, I. C. B. (2002). R-1 Teste não verbal de inteligência: manual de Rynaldo de Oliveira. São Paulo: Vetor.

AERA - American Educational Research Association, APA - American Psychological Association, NCME - National Council on Measurement in Education (1999). Standards for educational and psychological testing. Washington, DC: American Educational Research Association. Disponível on-line em: http://www.intestcom.org.

Angelini, A. L., Alves, I. C. B., Custódio, E. M., Duarte, W. F., \& Duarte, J. L. M. (1999). Matrizes Progressivas Coloridas de Raven-Escala Especial. São Paulo: Centro Editor de Testes e Pesquisa em Psicologia.

Arbuckle, J. L. (2009). Amos 18 user's guide. Chicago, IL: SPSS Inc.

Bentler. P. M., \& Bonett, D. G. (1980). Significance tests and goodness-of-fit in the analysis of covariance structures. Psychological Bulletin, 88, 588-606.

Bock, R. D., Gibbons, R., \& Muraki, E. (1988). Full information item factor analysis. Applied Psychological Measurement, 12(3), 261-280.

Carrol, J. B. (1997). The Three-Stratum Theory of Cognitive Abilities. Em D. P. Flanagan, J. L. Gensheft, \& P. L. Harrison, (Eds), Contemporany Intelectual Assesment: theories, tests and issues. (pp.122-130). Nova Iorque, London: The Guilford Press.

Flanagan, D. P., \& Ortiz, S. O. (2001). Essentials of cross-battery assessment. Nova Iorque: John Wiley \& Sons, Inc.

Gazzaniga, M. S., Ivry, R. B., \& Mangun, G. R. (2002). Cognitive neuroscience: the biology of the mind. Nova Iorque: Norton \& Company.

Goel, V. (2005). Cognitive neuroscienceof deductive reasoning. Em K. J. Holyoak \& R. G. Morrison (Eds.), The Cambridge handbook of thinking and reasoning (pp. 475-492). Nova Iorque: Cambridge.

Holyoak, K. J., \& Morrison, R. G. (2005). Thinking and reasoning: a reader's guide. Em K. J. Holyoak \&
R. G. Morrison (Eds.), The Cambridge Handbook of Thinking and Reasoning (pp. 1-9). Nova Iorque: Cambridge.

Hunt, E. (1999). Intelligence and human resources: past, present and future. Em P. L. Ackerman, P. C. Kyllonen, \& R. D. Roberts (Eds.), Learning and individual differences: process, trait and content determinants. Washington, DC: American Psychological Association.

Lezak, M.D. (1995). Neuropsychological assessment. Nova Iorque, NY: Oxford Univ Press.

McGrew, K. S. (2009). CHC theory and the human cognitive abilities project: standing on the shoulders of the giants of psychometric intelligence research. Intelligence, 37, 1-10.

Muniz, M. (2013). Inteligência no processo de ensino aprendizagem. Capítulo em preparação.

Muthén, L. K., \& Muthén, B. O. (2004). Mplus: the comprehensive modeling program for applied research. Los Angeles: Muthén \& Muthén.

Pasquali, L., Wechsler, S., \& Bensusan, E. (2002). Matrizes Progressivas do Raven Infantil: um estudo de validação para o Brasil. Avaliação Psicológica, 2, 95-110.

Posner, M. I., \& DiGirolamo, G. J (2000). Cognitive neuroscience: origins and promise. Psychological Bulletin, 126(6), 873-889.

Primi, R., \& Almeida, L. (1998). Considerações sobre a análise fatorial de itens com resposta dicotómica. Psicologia Teoria Investigação e Prática, 3, 225-234.

Raven J. C. (2000). Matrizes Progressivas: escala geral. Rio de Janeiro: CEPA.

Rueda, F. R. M., \& Castro, N. R. (2012). Teste de Inteligência-TI. São Paulo: Vetor.

Schneider, J., \& McGrew, K. (2011). Cattell-Horn-Carroll (CHC) Theory of Cognitive Abilities Definitions. (CHC v2.0) [On-Line]. Disponível em http://www.iapsych. com

Sisto, F. F. (2006). Teste de Raciocínio Inferencial: manual técnico. São Paulo: Vetor.

Sisto, F. F., Rueda, F. J. M., \& Bartholomeu, D. (2006). Estudo sobre a unidimensionalidade do teste $\mathrm{Ma}$ trizes Progressivas Coloridas de Raven. Psicologia: Reflexão \& Crítica, 19(1), 66-73. 
Sisto, F. F., Santos, A. A. A., \& Noronha, A. P. P. (2007). Estudo sobre a dimensionalidade do Teste R1-Forma B. Psicologia em Estudo, 12(1), 185-193.

Spearman, C. (1927). Las habilidades del hombre: su naturaleza y medición. Buenos Aires: Paidos.

Spearman, C. (1904). "General Intelligence", objectively determined and measured. American Journal of Psychology, 15, 201-293.
Sternberg, R. J. (1986). Toward a unified theory of human reasoning. Intelligence, 10, 281-314.

Tabachnick, B. G., \& Fidell, L. S. (2001). Using multivariate statistics. Needham Heights, MA: Allyn \& Bacon.

Thurstone, L. L. (1934). The vetors of mind. Psychological Review, 41, 1-32.

Recebido em: 06/05/2013

Reformulado em: $12 / 11 / 2013$

Aprovado em: 02/12/2013

Sobre os autores:

Fabián Javier Marín Rueda é psicólogo, mestre e doutor em Psicologia pela Universidade São Francisco. É docente da graduação e do Programa de Pós-Graduação Stricto Sensu em Psicologia da Universidade São Francisco.

Monalisa Muniz Nascimento é psicóloga, mestre e doutora em Psicologia. Atualmente é professora adjunta do Departamento de Psicologia da Universidade Federal de São Carlos - UFSCar.

Contato com os autores:

Universidade São Francisco,

Faculdade de Ciências Humanas, U S F.

Rua Alexandre Rodrigues Barbosa, 45 / Centro

CEP: 13251-900 - Itatiba, SP - Brasil

E-mail: marinfabian@yahoo.com.br 